\title{
Michele Muggeo
}

\author{
Enzo Bonora ${ }^{1} \cdot$ Riccardo Bonadonna ${ }^{2} \cdot$ Paolo Moghetti ${ }^{1}$
}

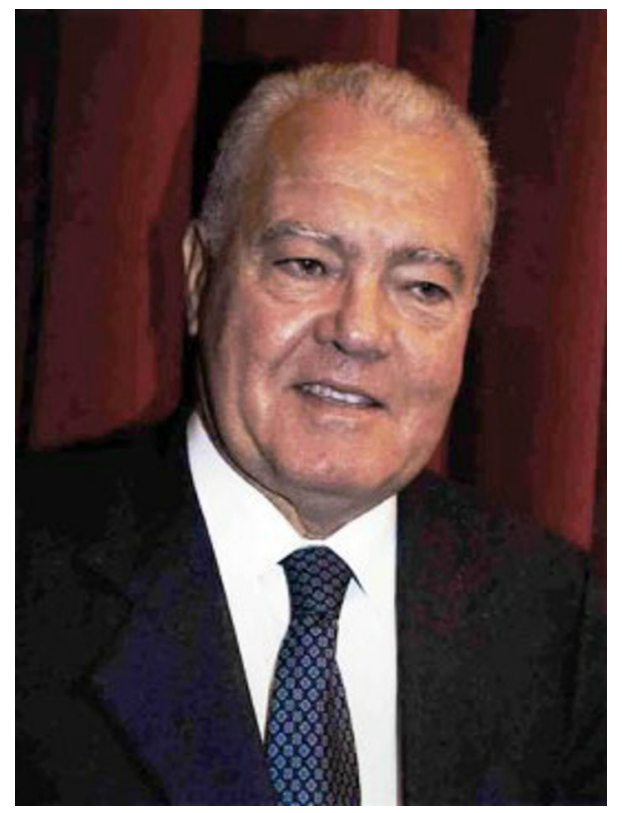

Nella notte fra il 22 e il 23 agosto 2021, all'età di 82 anni, si è spento serenamente nella sua abitazione di Padova, accanto alla sua amata moglie Maria, il prof. Michele Muggeo, fondatore della Scuola Endocrino-Metabolica di Verona e nostro illuminato Maestro.

Nato a Barletta nel 1938, il prof. Muggeo si è laureato a Padova nel 1964, formandosi e crescendo professionalmente nella Scuola dei Professori Patrassi e Crepaldi, dapprima

$凶$ E. Bonora

enzo.bonora@univr.it

1 Divisione of Endocrinologia, Diabete e Metabolismo, Dipartimento di Medicina, Università di Verona, Verona, Italia

2 Dipartimento di Medicina e Chirurgia, Università di Parma, Parma, Italia come medico interno e poi come assistente volontario, assistente di ruolo e, infine, come aiuto universitario. Negli anni Sessanta e Settanta ha acquisito ben sei specializzazioni, nell'ordine Ematologia, Cardiologia, Medicina Interna, Gerontologia e Geriatria, Endocrinologia, Diabetologia e Malattie del Ricambio, e, giovanissimo, una libera docenza in Semeiotica Medica. Nella seconda metà degli anni Settanta ha trascorso un lungo periodo di studio e ricerca come Visiting Scientist presso la Diabetes Branch dell'NIH di Bethesda, MD, USA, a fianco di ricercatori del calibro di Jesse Roth, Ronald Kahn, Philip Gorden, Jeffrey Flier, Robert Bar, Emmanuel Van Obberghen, Pierre De Meyts, Len Harrison, Barry Ginsberg. Nel 1980 è stato chiamato come Professore Ordinario di Endocrinologia dall'Università di Ancona e due anni dopo dall'Università di Verona, dove ha operato per quasi trent'anni, fino alla quiescenza nel 2009, essendone poi nominato Professore Emerito. Negli anni trascorsi presso l'Università di Verona ha diretto dapprima la Scuola di Specializzazione in Diabetologia e Malattie del Ricambio e la Scuola di Specializzazione in Endocrinologia e, dal 1991, la Scuola di Specializzazione in Endocrinologia e Malattie del Metabolismo. Ha anche coordinato il Dottorato di Ricerca in Malattie Cronico-Degenerative e in seguito quello in Scienze Mediche Cliniche e Sperimentali. Per oltre 25 anni è stato direttore del Centro di Verona del Gruppo di Studio delle Malattie Dismetaboliche e dell'Aterosclerosi.

La sua attività scientifica ha spaziato da studi sulla fisiopatologia della secrezione del GH a pioneristiche ricerche sul recettore insulinico, da studi sulla fisiopatologia dell'insulinoresistenza a ricerche sull'epidemiologia del diabete e delle sue complicanze. Numerosi i contributi derivati dal Verona Diabetes Study che lui ha ideato e guidato per anni. I suoi studi hanno portato alla pubblicazione di oltre 300 lavori, di cui oltre la metà su prestigiose riviste internazionali. Ha anche contribuito alla stesura di capitoli di numerosi trattati 
e atti di congressi nazionali e internazionali. Ha fatto parte dell'Editorial Board di numerose riviste e per moltissime riviste ha esercitato la funzione di revisore. La sua ricerca è stata finanziata dal Ministero dell'Università, dal Ministero della Salute, dal CNR e da varie altre istituzioni nazionali e internazionali.

Il prof. Muggeo è stato membro attivissimo della Società Italiana di Diabetologia (SID), svolgendo vari compiti, fra cui il coordinamento del Gruppo di Studio per l'Educazione (GISED, 1981-1991). È stato per due quadrienni membro del consiglio direttivo SID (1986-1990 e 1998-2002) e nel biennio 2000-2002 ne è stato Presidente, organizzandone il Congresso Nazionale a Verona nel 2002. La SID l'ha successivamente nominato Presidente Onorario. È stato anche componente del Consiglio Direttivo della Società Italiana di Endocrinologia (SIE) nel quadriennio 1982-1986, membro della Commissione designata a elaborare il nuovo statuto della società nel 1985 e organizzatore di due congressi nazionali tenutisi a Verona nel 1988 e nel 2007. È stato anche membro di altre società scientifiche in Italia, oltre che della American Diabetes Association e della European Association for the Study of Diabetes, del cui gruppo di studio sull'Epidemiologia (EDEG) ha organizzato un convegno a Verona nel 1999. Qualche anno prima, sempre a Verona, aveva organizzato il $4^{\text {th }}$ International Symposium on Insulin Receptor and Insulin Action. Nel complesso, l'attività organizzativa di eventi scientifici locali, regionali, nazionali e internazionali è sempre stata intensa.

Michele Muggeo era una persona di un'onestà cristallina, incrollabilmente leale e sincero, generoso, disponibile, socievole e ospitale. Quanto era serio e impegnato nel lavoro, tanto diventava amicale, empatico e affettuoso nelle pause di lavoro e nella convivialità. Gli piaceva avere persone intorno a cui offrire qualcosa. Era un buono. Una brava persona.

Nel suo lavoro Michele Muggeo era appassionato, instancabile, meticoloso, tenace, lucido e lungimirante. Aveva un innato spirito di sacrificio, una inesauribile percezione del proprio dovere e un fortissimo senso di appartenenza. Fosse questa l'Università, l'Ospedale, la struttura che dirigeva, la comunità diabetologica ed endocrinologica italiana, la cerchia di amici e colleghi italiani e di oltre confine. È stato uno straordinario promotore dell'endocrinologia e della diabetologia italiana in tutte le loro espressioni: scientifiche, cliniche, formative, divulgative e ha alimentato spesso collaborazioni per far crescere i suoi ma anche gli altri. Molti, in vari contesti, sono maturati e cresciuti professionalmente grazie ai suoi consigli e alle sue ispirazioni.

Tuttavia, i tre aggettivi che a nostro avviso meglio lo descrivono nel contesto lavorativo sono visionario, trascinatore e costruttore.

Visionario, nel senso positivo del termine, perché pensava in grande e vedeva certe cose prima di altri, nel cam- po scientifico, didattico e clinico. Intuì, lui che aveva solide basi in campo molecolare e fisiopatologico, l'importanza dell'epidemiologia nel campo del diabete. Intuì la necessità di essere innovativi nella trasmissione della conoscenza agli altri, fossero questi specialisti, medici del territorio, infermieri, farmacisti o altre figure professionali, persone con il diabete. Intuì che certe patologie, come il piede diabetico o l'ipercolesterolemia familiare, meritavano più attenzione e compassione. Compassione che ebbe sempre in abbondanza, unitamente all'empatia, nella sua attività di medico.

Trascinatore perché quando individuava un obiettivo ti faceva complice nella passione per lo stesso, ti stimolava, ti faceva capire che era la scelta più appropriata anche quando tu eri perplesso e poi ti trascinava verso quell'obiettivo. Verso il successo che lui vedeva come un successo di gruppo più che suo personale.

Costruttore perché ha fondato dal nulla una scuola e una struttura assistenziale che hanno una collocazione di prestigio nel panorama nazionale e internazionale. Quando arrivò a Verona nel 1982 era solo e osteggiato dall'ambiente, percepito come un estraneo forse di passaggio. Non si è arreso mai di fronte a nulla, indomito, e poco a poco, anno dopo anno, è riuscito ad attrarre persone, a ottenere spazi e attrezzature e personale di ruolo. E quando non otteneva personale di ruolo, generava posti precari cercando, fino allo sfinimento, fondi per sostenerli, senza abbandonare mai nessuno. È riuscito a far crescere in maniera esponenziale quello che aveva fondato dal nulla senza aiuti politici o accademici particolari, senza compromessi di sorta, solo duro lavoro. Non si è mai vantato di questo, eppure, da uomo solo a cui era stata concessa una stanzetta di 15 metri quadrati e l'uso di una porzione di un bancone di laboratorio, in 25 anni ha costruito una struttura in cui lavorano quasi 100 persone, producendo scienza, formazione e assistenza come in poche altre sedi in Italia. Tutto perché lui nel 1982 aveva guardato oltre, gettato le fondamenta e lavorato alacremente per realizzare quello che allora sembrava un sogno impossibile. Lui, il visionario, il trascinatore, il costruttore.

Questo è stato e per noi sempre sarà Michele Muggeo: un vero e inarrivabile Maestro nella ricerca, nella didattica e nella clinica. Non è stato però solo questo per noi, che per molti anni abbiamo avuto il privilegio di lavorare al suo fianco. È stato anche un formidabile esempio di grande umanità e dell'arte di saper cogliere nel quotidiano motivi per sorridere o per entusiasmarsi. A lui andrà sempre il nostro pensiero affettuoso e riconoscente.

Enzo Bonora, Riccardo Bonadonna e Paolo Moghetti a nome di tutti gli allievi.

Nota della casa editrice Springer Nature rimane neutrale in riguardo alle rivendicazioni giurisdizionali nelle mappe pubblicate e nelle affiliazioni istituzionali. 